\title{
The logarithmic equation of state for superconducting cosmic strings
}

\author{
Betti Hartmann 1 \\ School of Engineering and Science, Jacobs University Bremen, 28759 Bremen, Germany \\ Brandon Carter 2 \\ LUTh, Observatoire de Paris, Meudon, France
}

1st November 2008

\begin{abstract}
This investigation follows up the suggestion that the equation of state for superconducting cosmic strings provided by Witten's prototype biscalar field model can be well represented by an effective Lagrangian of simple logarithmic form depending on only 3 independent parameters. The numerical work described here confirms the validity of this approximation, and initiates the evaluation of the 3 required parameters, as functions of the masses and other parameters specifying the underlying $\mathrm{U}(1) \times \mathrm{U}(1)$ scalar field model in the limit for which the relevant gauge coupling constants are small.In this limit, subject to calibration of the relevant length and mass scales, the scalar field model is characterised by just 3 dimensionless ratios which (in order to provide conducting strings) must be subject to three inequalities (of which two have obvious analytic expressions). It is found here that when all three of these inequalities are satisfied by a reasonably large margin, there is a simple empirical formula that can be used to provide a fairly accurate prescription for the algebraic dependence on these 3 dimensionless ratios of the 3 parameters required for the logarithmic equation of state.
\end{abstract}

\footnotetext{
${ }^{1}$ E-mail:b.hartmann@jacobs-university.de

${ }^{2}$ E-mail:brandon.carter@obspm.fr
} 


\section{Introduction}

This work is a contribution to a program whose purpose is to provide appropriate equations of state - expressible as prescriptions for an appropriate Lagrangian action density on the world sheet - for conducting string models of the kind needed for description of Witten's bosonic superconducting string mechanism [1], of which a potentially important consequence [2] is the formation of vortons.

As a generalisation of the Nambu Goto prescription, which is simply a constant, Witten originally suggested [1] that (in the absence of electromagnetic background fields) as a function of the quadratic norm, $w=\varphi_{i} \varphi^{i}$, of the surface gradient with components $\varphi_{i}=\varphi_{, i}$ of the relevant scalar phase field $\varphi$, the action density on the 2-dimensional string worldsheet might be taken to have the linear form

$$
L=-m^{2}-\frac{1}{2} \kappa_{0} w
$$

depending on just two constant parameters $m$ (interpretable as a Kibble mass scale) and $\kappa_{0}$. It is evident that such a simplification can not provide a description of the effect of saturation when the current is large. However a more serious weakness is that even for the weak current limit in which $w$ is very small such a linear expression can not provide a satisfactory description of the propagation of small longitudinal perturbations, whose velocity depends not just on the first but also on the second derivative of $L$ with respect to $w$.

To provide an acceptable description of perturbations in the weak current limit, as well as of what has been found [3] to occur when the current is larger, it has been proposed that, rather than the Lagrangian itself, it is the inverse of the derived quantity

$$
\kappa=-2 \frac{\mathrm{d} L}{\mathrm{~d} w}
$$

that should be taken to be linear. This means that the Witten formula (11) should be replaced by an expression of the logarithmic form

$$
L=-m^{2}-\frac{1}{2} m_{\star}^{2} \ln \left\{1+\delta_{\star}^{2} w\right\},
$$

which depends on three fixed parameters, of which one is the Kibble mass scale, $m$ (as before) while the others are a secondary mass scale $m_{\star}$ and a lengthscale $\delta_{\star}$, whose squared product is identifiable with the original Witten 
model parameter, $\kappa_{0}$. The latter is interpretable as the zero current value of the quantity (2) which for non zero values of $w$ will be given by

$$
\kappa=\frac{\kappa_{0}}{1+\delta_{\star}^{2} w}, \quad \kappa_{0}=m_{\star}^{2} \delta_{\star}^{2} .
$$

Models of this kind are a special subclass within the general category needed for studying properties of generic conducting string models, of which an important example is the analysis [5, 6, 7, 8] of the stability of their vorton (closed loop) equilibrium states. Whether the particular logarithmic form (3) will be able to provide a good description for conducting string defects in realistic field models such as those of the standard electroweak theory and its various extensions [9, 10] is a problem that will be left for future work. The present article will be concerned just with its application in the restricted class of toy $\mathrm{U}(1) \times \mathrm{U}(1)$ field models proposed in this context by Witten.

Our purpose here is thus to investigate the dependence of the three parameters $m, m_{\star}^{2}, \delta_{\star}^{2}$, in the logarithmic string model on the various parameters needed in Witten's class [1] of toy field models. It was already recognised in the earliest pioneering exploration of the relevant parameter space [11] that there will only be a restricted part of it for which the non-vanishing currents envisaged by Witten will actually be able to occur. Outside the parameter domain of this restricted Witten subclass, the ensuing string model will merely be of the Nambu Goto type, as obtained from the Witten formula (1) by setting $\kappa_{0}=0$, or equivalently from the logarithmic formula (3) by setting $m_{\star}=0$.

One of the main advances in the present work will be to provide a much more accurate and complete description of the conditions that are necessary and sufficient for the characterisation of this Witten domain within the entire parameter space of the Witten class of toy scalar field models. Near the boundaries of this domain, the parameter dependence of the quantities characterising the conducting string model turns out to be rather sensitive. However better behaviour is obtained whenever the necessary inequalities are all satisfied with a reasonably large margin, and in such generic circumstances it is found that simple algebraic formulae can be provided for the specification with reasonable accuracy of the empirical string model parameters $m, m_{\star}^{2}, \delta_{\star}^{2}$ as functions over the Witten domain in the parameter space of the underlying scalar field model. 


\section{Specification of the class of field models}

The Witten class of superconducting string models, to which the present work applies, is characterised by appropriate parameter restrictions within a more complete category that has recently been set up for a different purpose by Saffin [12, whose concern was with another part of the relevant parameter space where it gives rise to strings that are non-conducting but that are instead characterised by not just one but two independent winding numbers, a feature that gives rise to the possibility of string junctions [13, 14].

The complete $\mathrm{U}(1) \times \mathrm{U}(1)$ category [12] consists of Abelian models involving a pair of complex scalar fields $\Psi_{\mathbf{a}}$ with label $\mathbf{a}=1,2$ that are coupled by a potential $\mathcal{V}$ but subject to a Lagrangian of the (rationalised) form

$$
\mathcal{L}=-\sum_{\mathbf{a}}\left(\frac{1}{2}\left(\mathcal{D}_{\mu} \Psi_{\mathbf{a}}\right) \mathcal{D}^{\mu} \bar{\Psi}_{\mathbf{a}}+\frac{1}{4} F^{\mathbf{a}}{ }_{\mu \nu} F^{\mathbf{a} \mu \nu}\right)-\mathcal{V}
$$

in which the kinetic part is entirely decoupled, meaning that each of the scalar fields $\Psi_{\mathbf{a}}$ is subject to the gauge action only of its own corresponding Abelian connection $A_{\mu}^{\mathbf{a}}$ with corresponding field $F_{\mu \nu}^{\mathbf{a}}=2 \nabla_{[\mu} A_{\nu]}^{\mathbf{a}}$ according to the simple specification whereby each has its own charge coupling constant $e_{\mathbf{a}}$ in terms of which $\mathcal{D}_{\mu} \Psi_{\mathbf{a}}=\left(\nabla_{\mu} \Psi_{\mathbf{a}}-i e_{\mathbf{a}} A_{\mu}^{\mathbf{a}}\right) \Psi_{\mathbf{a}}$. Each complex scalar is expressible in the usual manner, $\Psi_{\mathbf{a}}=\psi_{\mathbf{a}} \exp \left\{i \varphi_{\mathbf{a}}\right\}$ in terms of a real phase angle $\varphi_{\mathbf{a}}$ and a real amplitude $\psi_{\mathbf{a}}$. Only the amplitudes are involved in the potential, which is taken to be given in terms of a pair of mass scales $\tilde{\eta}_{\mathbf{a}}$ by an expression of the quartic form

$$
\mathcal{V}=\frac{1}{4} \sum_{\mathbf{a b}} \lambda^{\mathbf{a b}}\left(\psi_{\mathbf{a}}^{2}-\tilde{\eta}_{\mathbf{a}}^{2}\right)\left(\psi_{\mathbf{b}}^{2}-\tilde{\eta}_{\mathbf{b}}^{2}\right)+\mathcal{V}_{0}
$$

in which the $\lambda^{\mathbf{a b}}$ are the three independent components of a symmetric matrix that is restricted by the condition that $\mathcal{V}$ must be bounded below, and $\mathcal{V}_{0}$ is a dynamically redundant constant included for the purpose of adjusting the lower bound of $\mathcal{V}$ to be zero. This condition evidently requires that the diagonal coefficients should both be positive $\lambda^{11} \geq 0, \quad \lambda^{22} \geq 0$. It can be seen that a further necessary and sufficient condition for the potential to be bounded below is that the off diagonal component should be subject to the lower bound $\lambda^{12} \geq-\sqrt{\lambda^{11} \lambda^{22}}$. Saffin's concern [12] was with the class characterised by positivity of the determinant $\lambda^{11} \lambda^{22}-\left(\lambda^{12}\right)^{2}$ itself, for which (with $\mathcal{V}_{0}=0$ ) the vacuum is characterised by simultaneously non-vanishing field values $\psi_{1}=\tilde{\eta}_{1}$ and $\psi_{2}=\tilde{\eta}_{2}$. 
It is the opposite alternative possibility with negative determinant, as specified by the more restrictive lower bound

$$
\lambda^{12}>\sqrt{\lambda^{11} \lambda^{22}}
$$

that characterises models of the Witten class with which we are concerned here. In this case the potential has minima where one or other of the fields vanish, specifically - setting $\eta_{1}^{2}=\tilde{\eta}_{1}^{2}+\lambda^{12} \tilde{\eta}_{2}^{2} / \lambda^{11}$ and $\eta_{2}=\tilde{\eta}_{2}^{2}+\lambda^{12} \tilde{\eta}_{1}^{2} / \lambda^{22}-$ where $\psi_{1}=0$ with $\psi_{2}=\eta_{2}$, and where $\psi_{2}=0$ with $\psi_{1}=\eta_{1}$. The vacuum state itself (excluding the degenerate case for which the minima are equal, which gives a model with domain walls) will be the one for which $\mathcal{V}$ is lowest. Without loss of generality this can be taken to be the latter, where $\psi_{\mathbf{2}}=0$, by choosing the labelling so that the new mass scales satisfy

$$
\lambda^{11} \eta_{1}^{4}>\lambda^{22} \eta_{2}^{4}
$$

rather than the other way round. With his convention we shall be left with

$$
\mathcal{V}=\frac{\lambda^{11}}{4}\left(\psi_{\mathbf{1}}^{2}-\eta_{\mathbf{1}}^{2}\right)^{2}+\frac{\lambda^{12}}{2} \psi_{\mathbf{1}}^{2} \psi_{\mathbf{2}}^{2}+\frac{\lambda^{22}}{4} \psi_{\mathbf{2}}^{2}\left(\psi_{\mathbf{2}}^{2}-2 \eta_{\mathbf{2}}^{2}\right)
$$

after suitable adjustment of the additive constant The effective masses, $m_{1}$ and $m_{2}$ say, of the primary (Higgs type) and secondary (carrier) fields (namely $\Psi_{1}$ and $\Psi_{2}$ ) will then be given respectively by the formulae

$$
m_{1}^{2}=\lambda^{11} \eta_{1}^{2}, \quad m_{2}^{2}=\lambda^{12} \eta_{1}^{2}-\lambda^{22} \eta_{2}^{2},
$$

the requisite positivity of the latter being guaranteed as an automatic consequence of the inequalities (7) and (8).

The lengthscale

$$
\delta=\frac{1}{m_{1}}=\frac{1}{\eta_{1} \sqrt{\lambda^{11}}}
$$

associated with the primary field will roughly characterise the effective core radius of the ensuing string type vacuum defects. In order for such string defects to be strictly local (with finite world sheet energy density) we now follow Peter [3] in restricting our attention cases in which there is no coupling to any external electromagnetic field by setting $e_{\mathbf{2}}=0$. Far outside a string defect the field configuration will then converge towards the vacuum with an exponential cut off lengthscale $\ell$ (of the kind known in the context of ordinary metallic superconductivity as the London length) given in terms of 
the coupling constant $e_{1}$ of the other (non-electromagnetic) gauge field by the formula

$$
\ell^{2}=\frac{1}{e_{1}^{2} \eta_{1}^{2}}
$$

The well known Bogomolny limit condition - in the case for which the carrier field is absent - is simply that these lengthscales be equal $\ell=\delta$, which occurs when the coupling has the critical value $e_{1}^{2}=2 \lambda^{11}$. However our concern here will be with the weak coupling limit within the type II range characterised by the condition $\ell>\delta$. It is convenient to write

$$
\alpha=\sqrt{\frac{e_{1}^{2}}{\lambda^{11}}},
$$

so as to define a positive dimensionless charge coupling constant $\alpha$ in terms of which this type II range is characterised by the condition $\alpha<\sqrt{2}$. In addition to this dimensionless parameter $\alpha$, the system will be fully characterised qualitative, meaning modulo rescaling, by three other dimensionless parameters, which can be conveniently taken to be the mass ratio

$$
q=\frac{\eta_{2}}{\eta_{1}}
$$

and the pair of ratios

$$
\gamma_{2}=\frac{\lambda^{22}}{\lambda^{11}}, \quad \gamma_{3}=\frac{\lambda^{12}}{\lambda^{11}}
$$

In terms of these, the necessary (but as we shall see, not quite sufficient) inequalities (7) and (8) characterising the Witten subclass can be written conjointly as the condition

$$
q^{4} \gamma_{2}^{2}<\gamma_{2}<\gamma_{3}^{2}
$$

\section{Cylindrical vortex configurations}

The string model for a vortex defect of the vacuum is based on the assumption that a small segment thereof can be represented by a stationary cylindrically symmetric configuration in which, in terms of constants $\omega, k, n$, 
of which the latter is an integer winding number, the fields will be specified with respect to cylindrical coordinates $\{t, r, \theta, z\}$ by the ansatz

$$
e_{1} A_{\mu} \mathrm{d} x^{\mu}=(n-P) \mathrm{d} \theta, \Psi_{1}=\eta_{1} h \exp \{i n \theta\}, \Psi_{2}=\eta_{1} f \exp \{i k z-i \omega t\}
$$

in which the quantities $P, h, f$ depend only on the radial coordinate $r$, and are subject to the boundary conditions

$$
\begin{aligned}
& P \rightarrow n, \quad h \rightarrow 1, \quad f \rightarrow 0, \quad \text { as } r \rightarrow \infty, \\
& P \rightarrow 0, \quad h \rightarrow 0, \quad \frac{\mathrm{d} f}{\mathrm{~d} r} \rightarrow 0, \quad \text { as } r \rightarrow \infty .
\end{aligned}
$$

The corresponding macroscopic string description, of the kind discussed in the introduction, will be obtained by taking

$$
L=2 \pi \int \mathcal{L} r \mathrm{~d} r, \quad \kappa=2 \pi \int \psi_{\mathbf{2}}^{2} r \mathrm{~d} r, \quad w=k^{2}-\omega^{2},
$$

subject to the field equations. Using a prime for differentiation with respect to the dimensionless radial coordinate defined in terms of the lengthscale (11) as

$$
x=m_{1} r,
$$

these field equations will be given by

$$
\begin{gathered}
\left(\frac{P^{\prime}}{x}\right)^{\prime}=\alpha^{2} \frac{P h^{2}}{x}, \\
\frac{1}{x}\left(x h^{\prime}\right)^{\prime}=\frac{P^{2} h}{x^{2}}+h\left(h^{2}-1\right)+\gamma_{3} f^{2} h, \\
\frac{1}{x}\left(x f^{\prime}\right)^{\prime}=\frac{w}{m_{1}^{2}} f+\gamma_{2} f\left(f^{2}-q^{2}\right)+\gamma_{3} f h^{2} .
\end{gathered}
$$

\section{Procedure for empirical matching of the equation of state}

Numerical application of the foregoing procedure can in principle provide an exact equation of state specifying the dependence of $L$ on $w$. The suggestion [4] that the simple logarithmic formula (3) can provide a good approximation to the exact result was motivated by the earlier analysis of Peter 
[3] which shows that a singularity will occur when the energy associated with the current reaches the threshold for creation of particles of the carrier field with the mass $m_{2}$ given by (10), which can be seen to occur when $w=-m_{2}^{2}$. On this basis, the appropriate value for the parameter $\delta_{\star}$ in the equation of state can be evaluated in advance simply as

$$
\delta_{\star}=\frac{1}{m_{2}}
$$

To complete the specification of the logarithmic formula (3) it remains only to obtain the mass parameters $m$ and $m_{\star}$ which will both be obtainable from knowledge just of the zero current limit, by evaluation of the corresponding values $L_{0}$ and $\kappa_{0}$ as given by the integral formulae (20) for $L$ and $\kappa$ when $w=0$, from which one will obtain

$$
m^{2}=-L_{0}, \quad m_{\star}^{2}=m_{2}^{2} \kappa_{0} .
$$

This case $w=0$ includes the strictly static configuration characterised by $\omega=0$ as well as $k=0$, for which the field equations will be obtainable just by minimisation of the corresponding energy integral. In view of the staticity the latter will be equal in magnitude but opposite in sign to the Lagrangian, which takes the form

$$
L_{0}=-\eta_{1}^{2} \tilde{U}, \quad \tilde{U}=2 \pi \int \tilde{\mathcal{E}} x \mathrm{~d} x,
$$

in which the dimensionless rescaled energy density is given by

$$
2 \tilde{\mathcal{E}}=\frac{P^{\prime 2}}{\alpha^{2} x^{2}}+\frac{P^{2} h^{2}}{x^{2}}+h^{\prime 2}+\frac{\left(h^{2}-1\right)^{2}}{2}+\gamma_{\mathbf{3}} h^{2} f^{2}+f^{\prime 2}+\gamma_{\mathbf{2}} \frac{f^{4}}{2}-\gamma_{\mathbf{2}} q^{2} f^{2} .
$$

Subject to the minimisation of the total energy integral $\tilde{U}$, the corresponding expression for the required condensate integral will be

$$
\kappa_{0}=2 \pi \frac{\eta_{1}^{2}}{m_{1}^{2}} \int f^{2} x \mathrm{~d} x .
$$

We thus end up with the integral prescriptions

$$
\frac{m^{2}}{\eta_{1}^{2}}=\tilde{U}, \quad \frac{m_{\star}^{2}}{\eta_{1}^{2}}=\left(\gamma_{\mathbf{3}}-\gamma_{\mathbf{2}} q^{2}\right) 2 \pi \int f^{2} x \mathrm{~d} x .
$$




\section{The third restriction}

It is to be noticed that all the terms in (28) are positive except the last, which is homogeneously quadratic in $f$, and that it is only where the magnitude of this last term exceeds that of the other terms quadratic in $f$ that local minimisation will leave a non vanishing value for this condensate amplitude $f$. In particular this will occur only where $\gamma_{3} h^{2}<\gamma_{2} q^{2}$, which for winding number $n=1$ means roughly where $\gamma_{3} x^{2} \lesssim \gamma_{2} q^{2}$, since it is to be expected that the order of magnitude of the primary field will be given roughly by $h \simeq 1$ for $x \gtrsim 1$ and $h \approx x$ for $x \lesssim 1$. The implication that non-vanishing values of $f$ must be roughly confined within the thin tube characterised by $x^{2} \lesssim q^{2} \gamma_{\mathbf{2}} / \gamma_{\mathbf{3}}$ entails that the squared fractional gradient of $f$ in the tube should on average satisfy $\left(f^{\prime} / f\right)^{2} \gtrsim \gamma_{3} /\left(\gamma_{2} q^{2}\right)$. However this conflicts with the requirement that the term $f^{\prime 2}$ in (28) should also be dominated by the final term, $-\gamma_{2} q^{2} f^{2}$, unless we have

$$
\gamma_{3} \lesssim \gamma_{2}^{2} q^{4}
$$

which, in view of (16) entails that we must also have $\gamma_{3} \gtrsim 1$.

The implication of this is that, in order for the secondary field to provide a non vanishing condensate, as measured by the sectional integral $\kappa_{0}$ given by (29), and thus as a prerequisite for string conductivity, the two inequalities in (16) are not sufficient. To fully characterise the required Witten subclass, they must be be supplemented by a third parameter restriction that will evidently be given roughly by (31) and that has been obtained in a more precise form by numerical work.

This third restriction may be expressed in conjunction with the other two of the form

$$
c_{3} \gamma_{3}<\gamma_{2}^{2} q^{4}<\gamma_{2}<\gamma_{3}^{2} .
$$

in which $c_{3}$ is a dimensionless quantity of order unity. Whereas we had expected that it might also depend weakly on $\gamma_{2}$ or on $q^{2}$ our numerical computations have shown that (in the weak gauge coupling limit considered here) the lower bound $c_{3}$ can be specified with high accuracy as a function only of $\gamma_{3}$. The result obtained for the weak dependence of $c_{3}$ on $\gamma_{3}$ is plotted in Fig,1, from which it can be seen that for large values of $\gamma_{3}$ it increases towards a value given roughly by $c_{3} \simeq 1.4$. 


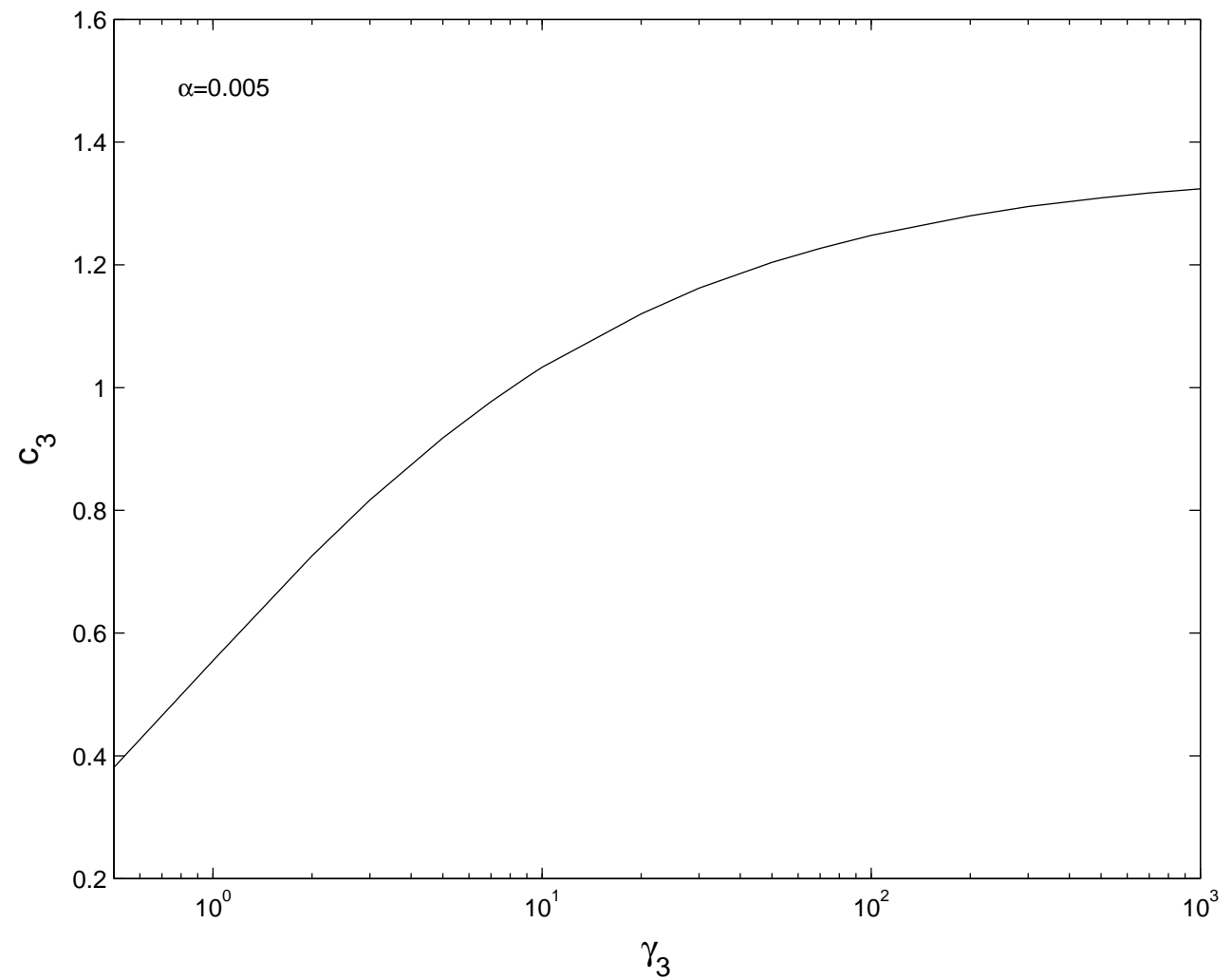

Figure 1: The value of $c_{3}$ (defined as the lower bound for $q^{4} \gamma_{2}^{2} / \gamma_{3}$ ) is plotted (for weak gauge coupling, $\alpha=0.005$ ) as a function of $\gamma_{3}$ over the allowed range of the latter, which must always be greater than about 0.3 . 


\section{Formula for the generic (non-marginal) case.}

Provided the conditions (31) are satisfied not just marginally but by a wide margin, the gradient term $f^{\prime 2}$ in (28) will be negligible so the contribution from $f$ will be effectively algebraic and thus minimisable locally, providing the approximation $f^{2} \simeq q^{2}-h^{2} \gamma_{3} / \gamma_{2}$ in the tube where this is positive, and $f^{2} \simeq 0$ outside. Again on the supposition that we shall have

$h \approx x$ inside the tube (whose radius will therefor be given by $x \approx q \sqrt{\gamma_{2} / \gamma_{3}}$ ) this provides the corresponding rough order of magnitude estimate

$$
\gamma_{3} \ll \gamma_{2}^{2} q^{4} \quad \Rightarrow \quad \int f^{2} x \mathrm{~d} x \approx \frac{q^{4} \gamma_{2}}{4 \gamma_{3}}
$$

for the integral needed in the formula (29) for the evaluation of $\kappa_{0}$.

The implication is that this quantity will be given by a formula of the form

$$
\kappa_{0}=c_{2} \frac{\gamma_{2} \eta_{1}^{2} q^{4}}{\gamma_{3} m_{1}^{2}}=c_{2} \frac{\lambda^{22} q^{2}}{\lambda^{11} \lambda^{12}},
$$

in which $c_{2}$ is a coefficient that will be non-zero only when the condition (31) is satisfied, and that will tend, when (31) is satisfied by a wide margin, towards a roughly constant order of unity value, $c_{2} \approx 1$.

In order to verify this, and to obtain a more precise estimate for the coefficient in (34) it is convenient to use this equation as a formal definition for the parameter $c_{\mathbf{2}}$, in terms of the quantity $\kappa_{0}$ given by (29) as the integral of $f^{2}$ for a chiral solution. On the basis of this definition, we have evaluated $c_{2}$ as a function of given $\gamma_{2}$, and $q^{2}$ for various fixed values of $\gamma_{3}$ taken well within the allowed range, that is for $\gamma_{3} \gg 1$. The results for the particular values $\gamma_{3}=30, \gamma_{3}=100$, and $\gamma_{3}=300$ are given in the three successive plots of Figure 2, which show contours for fixed values of the quantity $\gamma_{2} q^{4}$ at levels up to its marginal upper limit $\gamma_{2} q^{4}=1$. So long as $\gamma_{2} q^{4}$ is neither too close to this upper limit nor too near its lower bound (which decreases as $\gamma_{3}$ increases) it can be seen that over a wide range of $\gamma_{2}$ the value of $c_{2}$ is roughly constant with a value of the ratio $c_{2} /(2 \pi)$ that is close to unity, so that a reasonable approximation will be obtained by taking

$$
c_{2} \simeq 2 \pi \text {. }
$$

With $\delta_{\star}$ given in advance by (25), and with the parameter $\kappa_{0}$ - and hence also $m_{\star}^{2}=\kappa_{0} / \delta_{\star}^{2}$ - determined by the coefficient $c_{2}$, the only quantity still 

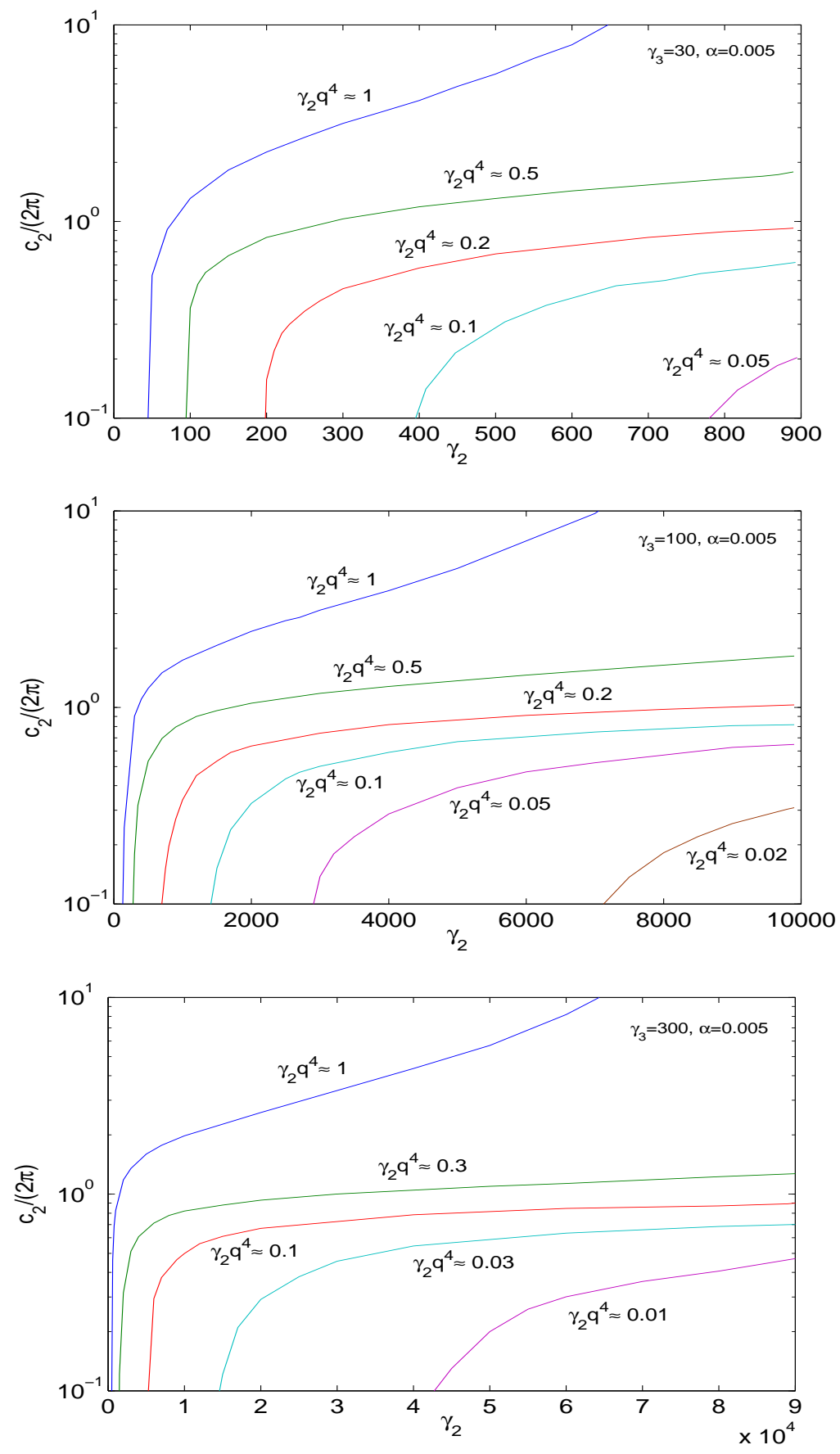

Figure 2: The value of $c_{\mathbf{2}} /(2 \pi)$ is plotted against $\gamma_{2}$ on chosen contours of constant $\gamma_{2} q^{4}$ (with $\alpha=0.005$ ) for the successive values 30, 100, 300 of $\gamma_{3}$. 


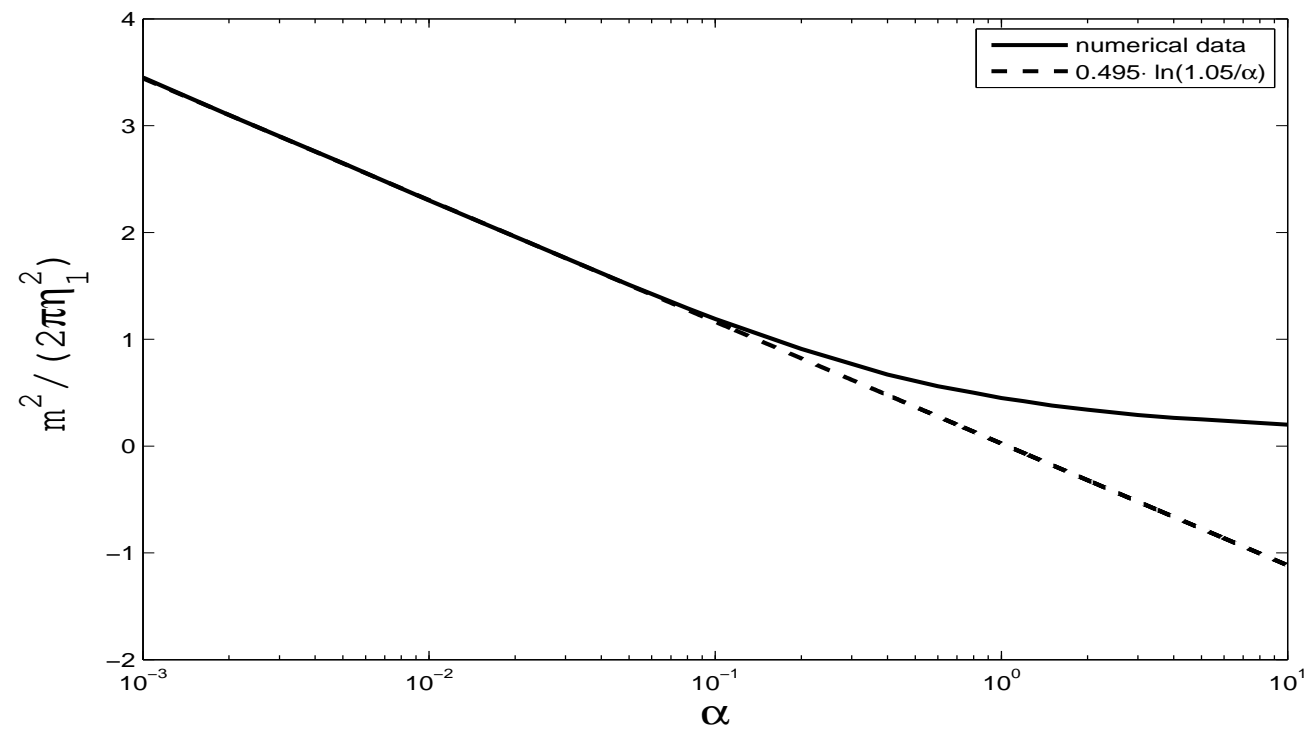

Figure 3: The value of $m^{2} /\left(2 \pi \eta_{1}^{2}\right)$ is plotted against the coupling constant $\alpha$, using a straight dashed line for the prediction of the equation (36) and a solid curve for the numerical data, which can be seen to fit the suggested form (36) very well for small $\alpha$ but to deviate - as expected - for $\alpha \gtrsim 0.1$.

needed for the complete specification of the logarithmic equation of state (3) is the value of the Kibble mass $m$ itself. For this, dimensional considerations suggest, that a reasonably good description will be provided by an expression of the form

$$
m^{2}=c_{1} \eta_{1}^{2} \ln \left\{\frac{c_{0}}{\alpha}\right\},
$$

in which, when the coupling is weak, $\alpha^{2} \ll 1$, the coefficients will have roughly constant order of unity values.

As shown by Figure 3, his expectation seems to be confirmed by our numerical computations which have given the values

$$
c_{1} \simeq 3.11, \quad c_{0} \simeq 1.05
$$




\section{Conclusions}

In this study of superconducting strings in the weakly coupled limit $(\alpha \ll 1)$ of the $\mathrm{U}(1) \times \mathrm{U}(1)$ field model of Witten, it has been found that in order to provide such conducting strings, the parameters characterising the field model must satisfy the necessary and sufficient conditions given by (32). The applicability, as a reasonably good approximation, of the previously suggested logarithmic form (3) for the effective Lagrangian characterising the equation of state has been confirmed. In terms of the parameters characterising the field model by the specification of the relevant potential (91), the required length scale $\delta_{\star}$ will be given by (25) and our numerical results (37) suggest that the relevant Kibble mass scale $m$ (which is all that is needed in the non-conducting Nambu Goto limit) may be taken with reasonable accuracy to be given, according to (36), by an ansatz of the easily memorable form

$$
m^{2}=\pi \eta_{1}^{2} \ln \left\{\frac{1}{\alpha}\right\}
$$

For the condensate integral $\kappa_{0}$ needed to complete the specification of the equation of state by providing the other required mass scale $m_{\star}$ using (4), the formula (34) provides a corresponding estimate

$$
\kappa_{0}=2 \pi \frac{\lambda^{22} q^{2}}{\lambda^{11} \lambda^{12}},
$$

that will be valid as a reasonable approximation when the conditions (32) are satisfied with a large margin, i.e. when

$$
\lambda^{11} \lambda^{12} \ll\left(\lambda^{22} q^{2}\right)^{2} \ll \lambda^{11} \lambda^{22} \ll\left(\lambda^{12}\right)^{2} .
$$

\section{Acknowledgments.}

We wish to thank X. Martin and M. Volkov for illuminating conversations. This work was supported by A.N.R prorgam NT05.1-42846. We also thank the ICTS visitors program of Jacobs University Bremen for financial support.

\section{References}

[1] E. Witten, "Superconducting strings", Nucl. Phys. B249 (1985) 557592. 
[2] R. Davis and P. Shellard, "Cosmic vortons", Nucl. Phys. B323 (1989) $209-224$.

[3] P. Peter, "Superconducting cosmic string: Equation of state for spacelike and timelike current in the neutral limit", Phys. Rev. D45 (1992) 1091.

[4] B. Carter and P. Peter "Supersonic string Model for Witten vortices", Phys. Rev. D52 (1995) 1744.

[5] B. Carter and X. Martin, "Dynamic instability criterion for circular string loops", Ann. Phys. 227 (1993) 151-171. arXiv:hep-th/0306111

[6] X. Martin, "Zones of dynamical instability for rotating string loops", Phys. Rev. D50 (1994) 7479-7492.

[7] X. Martin and P. Peter, "Dynamical stability of Witten vortons", Phys. Rev. D51 (1995) 4092-4098. arXiv:hep-ph/9405220]

[8] B. Carter, P. Peter and A. Gangui, "Avoidance of collapse by circular current-carrying cosmic string loops", Phys. Rev. D55 (1997) 4647-4662. arXiv:hep-ph/9609401

[9] A-C. Davis, W. Perkins, "Generic current-carrying strings", Phys. Lett. B390 (1997) 107-114. arXiv:hep-ph/9610292

[10] M.S. Volkov, "Superconducting electroweak strings", Phys. Lett. B644 (2006) 203-207. arXiv:hep-th/0609112

[11] A. Babul, T. Piran, D.N. Spergel, "Bosonic superconducting strings. 1. Classical field theory solutions", Phys. Lett. B202 (1988) 307-314.

[12] P.M. Saffin, "A practical model for cosmic (p,q) superstrings", J. High Energy Phys. 09 (2005) 011. arXiv:hep-th/0506138

[13] E.J. Copeland, P.M. Saffin, "On the evolution of multi string networks", J. High Energy Phys. 11 (2005) 023. arXiv:hep-th/0505110

[14] E.J. Copeland, T.W.B. Kibble, D.A. Steer, "Constraints on string networks with junctions", Phys. Rev. D75 (2007) 065024. arXiv:hep-th/0611243 